\title{
Cramér-Rao Bound Analysis of Localization Using Signal Strength Difference as Location Fingerprint
}

\author{
A.K.M. Mahtab Hossain and Wee-Seng Soh \\ Department of Electrical \& Computer Engineering \\ National University of Singapore, Singapore \\ Email: \{g0500774, elesohws\}@nus.edu.sg
}

\begin{abstract}
In this paper, we analyze the Cramér-Rao Lower Bound (CRLB) of localization using Signal Strength Difference (SSD) as location fingerprint. This analysis has a dual purpose. Firstly, the properties of the bound on localization error may help in designing efficient localization algorithms. For example, utilizing one of the properties, we propose a way to define weights for a weighted $\mathcal{K}$-Nearest Neighbor $(\mathcal{K}-\mathrm{NN})$ scheme which is shown to perform better than the $\mathcal{K}-\mathrm{NN}$ algorithm. Secondly, it provides suggestions for a positioning system design by revealing error trends associated with the system deployment. In both cases, detailed analysis as well as experimental results are presented in order to support our claims.

Keywords: Positioning Systems, Indoor Localization, Location Fingerprint, Cramér-Rao Lower Bound, Signal Strength.
\end{abstract}

\section{INTRODUCTION}

Recently, there has been a growing interest in indoor localization techniques that rely on in-building communications infrastructure (e.g., Wi-Fi, Bluetooth, etc.) mainly because it allows the design of an easily deployable low-cost positioning system. Most of these approaches utilize location fingerprinting techniques [1]-[4], where some location-dependent signal parameters are collected at a number of locations as location fingerprints in an "off-line training phase". During the "online location determination phase", the signal parameter obtained is compared with those training data to estimate the user location. The received signal strength (RSS) parameter has been the traditional choice for location fingerprint. However, it has been observed that, regardless of whether a mobile node's (MN's) signal strengths as perceived by the access points (APs) are used to denote the MN's location fingerprint (APbased localization), or the reverse approach in which the APs' signal strengths as perceived by the $\mathrm{MN}$ are used (MN-assisted localization), such fingerprints may differ significantly with the MN's hardware even under the same wireless conditions [5][8]. Most fingerprinting solutions use the same mobile device for both training and testing, thereby, shielding the adverse effect of this phenomenon. Rather than using RSS as location fingerprint, we proposed in [6] to use the difference of signal strengths perceived at the APs (for AP-based localization) or at the $\mathrm{MN}$ (for $\mathrm{MN}$-assisted localization) as the location fingerprint. We believe that the signal strength difference (SSD) is the ideal choice for location fingerprint if the positioning system administrator intends to accommodate heterogeneous devices. However, the error bound on localization using SSD has not been investigated yet. We feel that the properties of this bound could provide valuable insights to improving the localization accuracy or to the overall design of a positioning system based on SSD.

In this paper, we analyze the Cramér-Rao Lower Bound (CRLB) [9] of location estimation error given the SSD measurements. A novel characterization of the properties of this bound is presented that allows us to individually assess the impact of different parameters (e.g., number of APs, geometry of the APs, distance of the APs from the MN, etc.) on the accuracy of location estimates. For example, utilizing the effect of distances of the APs from the MN, we have devised a way to define weights for a weighted $\mathcal{K}$-NN scheme that is shown to perform better than the $\mathcal{K}-\mathrm{NN}$ algorithm. Moreover, the properties also provide valuable design phase suggestions by revealing error trends associated with the system deployment. We also investigate these deployment issues which may give fruitful insights into the design of a location system.

The study of estimation bounds on localization using time-of-arrival [10], time-difference-of-arrival [11], angle-ofarrival [11] techniques, or the RSS location fingerprint [12][14] have been investigated in the literature before. The findings subsequently opened the door for further analysis and design of various efficient localization algorithms which improve the accuracy [11], [13], [15]. We expect similar trend to follow for the SSD location fingerprint as well.

The rest of the paper is organized as follows. In Section II, we provide a brief review of our SSD location fingerprint, and discuss our localization algorithm in Section III. The CRLB analysis and the impacts of various properties of this bound are presented in detail in Section IV. Finally, we present in Section $\mathrm{V}$ the conclusions drawn.

\section{REVIEW OF SSD LOCATION FINGERPRINT}

The SSD fingerprint is shown to be robust across different mobile devices compared to the traditional RSS both analytically [6] and experimentally [5], [6]. Using the shadowing model, the SSD's expression can be obtained as in [6],

$$
\left[\frac{p_{k}}{p_{r}}\right]_{\mathrm{dB}}=-10 \beta \log \left(\frac{d_{k}}{d_{r}}\right)+\left[X_{k}-X_{r}\right]_{\mathrm{dB}},
$$

where $\beta$ is the path-loss exponent, $X_{k} \sim N\left(0, \sigma_{k}^{2}\right)$ and $X_{r} \sim N\left(0, \sigma_{r}^{2}\right)$ are the shadowing variations, $p_{k}$ and $p_{r}$ denote 
the RSSs at the MN from the $k^{\text {th }}$ and $r^{\text {th }}$ APs, which are at distances $d_{k}$ and $d_{r}$ from the $\mathrm{MN}$, respectively.

If a positioning system has $K$ APs, there can be $\left(\begin{array}{c}K \\ 2\end{array}\right)$ possible SSDs among which only $(K-1)$ values are independent. We obtain these SSD values as, $\left[\frac{p_{k}}{p_{r}}\right]_{\mathrm{dB}}, k=$ $\{1,2, \ldots, K\}-\{r\}$, where the $r^{\text {th }}$ AP is considered as the reference AP. The RSS of the $r^{\text {th }}$ AP is subtracted from the other $(K-1)$ APs' RSS values to produce the desired SSDs. An intuition about how we select the reference $r^{\text {th }}$ AP is given at the end of next section. However, for ease of our mathematical calculations in this paper, we assume the $K^{\text {th }}$ AP to be the reference AP, i.e., $r=K$.

\section{LOCALIZATION ALGORITHM}

First, we briefly discuss the traditional RSS-based Bayesian inference scheme to locate a user. Our SSD-based localization algorithm is just a slight modification on that scheme.

\section{A. Review of RSS-based Localization Algorithm}

We consider our indoor environment to be modeled as a finite position space $\left\{c_{1}, c_{2}, \ldots, c_{L}\right\}$ with a finite observation space $\left\{o_{1}, o_{2}, \ldots, o_{M}\right\}$. We define an observation as a vector of signal strength readings over $K$ APs, i.e., $o_{j}=\left\{p_{1}, p_{2}, \ldots, p_{K}\right\}$, where $p_{k}$ denotes the received signal strength from the $k^{\text {th }}$ AP. Based on different APs' signal strengths' independence assumption, we then define the conditional probability as, $\operatorname{Pr}\left(o_{j} \mid c_{i}\right)=\prod_{k=1}^{K} \operatorname{Pr}\left(p_{k} \mid c_{i}\right)$. After the training phase, for any given location $c_{i}$, we have a probability distribution $\operatorname{Pr}\left(o_{j} \mid c_{i}\right)$ that assigns a probability for each measured signal vector $o_{j}$. Subsequently, we obtain the posterior distribution of location by applying the Bayes' rule:

$$
\operatorname{Pr}\left(c_{i} \mid o_{j}\right)=\frac{\operatorname{Pr}\left(o_{j} \mid c_{i}\right) \operatorname{Pr}\left(c_{i}\right)}{\sum_{m=1}^{L} \operatorname{Pr}\left(o_{j} \mid c_{m}\right) \operatorname{Pr}\left(c_{m}\right)},
$$

where $\operatorname{Pr}\left(c_{i}\right)$ denotes the prior probability of being at location $c_{i}$. Without any background information such as user profile, it can be assumed to be uniformly distributed, i.e., $\frac{1}{L}$. As the denominator $\sum_{m=1}^{L} \operatorname{Pr}\left(o_{j} \mid c_{m}\right) \operatorname{Pr}\left(c_{m}\right)$ does not depend upon the location variable $i$, it can be safely treated as a normalizing constant whenever only relative probabilities or probability ratios are required.

During the testing phase, for an observation vector $o_{j}$, the conditional probability $\operatorname{Pr}\left(o_{j} \mid c_{i}\right)$ of each training location $c_{i}$ is calculated utilizing the offline data. Some existing works (e.g., [2], [4]) pick the position with the largest $\operatorname{Pr}\left(o_{j} \mid c_{i}\right)$ as the location estimate (Maximum Likelihood), while others (e.g., [6]) may choose the average of the $\mathcal{K}$ locations with largest $\operatorname{Pr}\left(o_{j} \mid c_{i}\right)$ 's to be the final estimate $(\mathcal{K}$-Nearest Neighbors).

\section{B. SSD-based Localization Algorithm}

Our SSD-based localization scheme is quite similar to the RSS-based algorithm discussed in the previous section. However, the observation vector is now a $(K-1)$-dimensional signal strength difference readings of the form, $o_{j}=\left\{p_{1}-\right.$ $\left.p_{r}, p_{2}-p_{r}, \ldots, p_{K-1}-p_{r}\right\}$ where $p_{r}$ denotes the reference $r^{\text {th }}$ AP's RSS. Subsequently, the conditional probability of the observation becomes, $\operatorname{Pr}\left(o_{j} \mid c_{i}\right)=\prod_{k=1}^{K-1} \operatorname{Pr}\left(p_{k}-p_{r} \mid c_{i}\right)$. Unless mentioned otherwise, we adopt the $\mathcal{K}$-Nearest Neighbors as our algorithm where the average of $\mathcal{K}$ locations having the largest $\operatorname{Pr}\left(o_{j} \mid c_{i}\right)$ 's gives the location estimate.

Now, let us discuss how we have modeled the conditional probability $\operatorname{Pr}\left(p_{k}-p_{r} \mid c_{i}\right)$. We assume the RSS from an AP at a particular location to be normally distributed. Though some works defy this phenomenon, others lend support to it [6], [16]. Similar to other works [17], we also have not observed any significant improvement when we consider the histogram representation of RSS compared to its Gaussian counterpart.

We post-process our training data to be fitted into the Gaussian distribution, $Z_{p_{k} \mid c_{i}} \sim N\left(\mu_{i k}, \sigma_{i k}^{2}\right)$, where $\mu_{i k}$ and $\sigma_{i k}$ being the average and standard deviation of the signal strength samples collected from the $k^{\text {th }}$ AP at training location $c_{i}$. Consequently, we obtain,

$$
Z_{p_{k}-p_{r} \mid c_{i}} \sim N\left(\mu_{i k}-\mu_{i r}, \sigma_{i k}^{2}+\sigma_{i r}^{2}\right) .
$$

We select the reference $r^{\text {th }}$ AP as the one which shows the least average deviation of RSSs over the whole localization area, i.e., $r=\min _{k}\left\{\frac{1}{L} \sum_{i=1}^{L} \sigma_{i k}^{2}\right\}, \forall k \in\{1,2, \ldots, K\}$.

\section{CRLB FOR LOCALIZATION USING SSD}

It is well-known that the Cramér-Rao Lower Bound (CRLB) sets a lower limit for the variance (or covariance matrix) of any unbiased estimates of an unknown parameter (or unknown parameters) [9]. If $\widehat{\theta}=(\widehat{x} \widehat{y})^{T}$ is the estimate of the MN's location $\theta=\left(\begin{array}{ll}x y \\ { }^{T}\end{array}{ }^{T}\right.$, then its covariance matrix is,

$$
\begin{aligned}
\operatorname{Cov}_{\theta}(\widehat{\theta}) & =E_{\theta}\left\{(\widehat{\theta}-\theta)(\widehat{\theta}-\theta)^{T}\right\} \\
& =\left[\begin{array}{cc}
\sigma_{\widehat{x}}^{2} & \sigma_{\widehat{x} \widehat{y}} \\
\sigma_{\widehat{y} \widehat{x}} & \sigma_{\widehat{y}}^{2}
\end{array}\right],
\end{aligned}
$$

where $E_{\theta}\{$.$\} is the expectation operator conditioned on \theta$. The diagonal elements of (3) represent the mean squared errors and the off-diagonal elements are the covariances between different parameters.

The lower bound is given in terms of the Fisher Information Matrix (FIM) [9]. If $f_{\theta}(P)$ denotes the probability density function (p.d.f.) of observations $P$ conditioned on $\theta$, then the score function [9] is defined as the gradient of its loglikelihood, i.e.,

$$
U(\theta)=\nabla \ln f_{\theta}(P)=\frac{\partial}{\partial \theta} \ln f_{\theta}(P)
$$

The FIM, $J(\theta)$ is the variance of this score function,

$$
J(\theta)=E\left\{\left[\frac{\partial \ln f_{\theta}(P)}{\partial \theta}\right]^{2}\right\} .
$$

If $f_{\theta}(P)$ belongs to some exponential family, then, with some regularity conditions imposed [9], we have,

$$
J(\theta)=-E\left\{\frac{\partial U(\theta)}{\partial \theta}\right\} .
$$

The CRLB is just the inverse of FIM and from its property,

$$
\operatorname{Cov}_{\theta}(\widehat{\theta}) \geq\{J(\theta)\}^{-1} \text {. }
$$


Utilizing SSD's expression in (1), the joint p.d.f. of the $(K-1)$ independent SSD measurements can be written as,

$$
\begin{aligned}
f_{\theta}(P)= & \prod_{k=1}^{K-1} \frac{1}{\sqrt{2 \pi} \widetilde{\sigma}} \frac{10}{\ln 10} \frac{p_{r}}{p_{k}} \times \\
& \exp \left\{-\frac{\left[10 \log \frac{p_{k}}{p_{r}}+10 \beta \log \left(\frac{d_{k}}{d_{r}}\right)\right]^{2}}{2 \widetilde{\sigma}^{2}}\right\},
\end{aligned}
$$

where $\tilde{\sigma}^{2}=\sigma_{k}^{2}+\sigma_{r}^{2}, p_{k}$ and $p_{r}$ are measured in $\mathrm{mW}$ scale, i.e., $p_{k}(\mathrm{dBm})=10 \log p_{k}$ and $d\left[\frac{p_{k}}{p_{r}}\right]_{\mathrm{dB}}=\frac{10}{\ln 10} \frac{p_{r}}{p_{k}} d\left[\frac{p_{k}}{p_{r}}\right]$. Let the FIM be denoted as,

$$
J(\theta)=\left[\begin{array}{ll}
J_{x x}(\theta) & J_{x y}(\theta) \\
J_{y x}(\theta) & J_{y y}(\theta)
\end{array}\right] .
$$

Using (7) into (4) and (5), the entries of (8) are obtained as,

$$
\begin{aligned}
J_{x x}(\theta) & =\rho \sum_{k=1}^{K-1}\left[\frac{\cos \phi_{k}}{d_{k}}-\frac{\cos \phi_{r}}{d_{r}}\right]^{2} . \\
J_{x y}(\theta) & =J_{y x}(\theta) \\
& =\rho \sum_{k=1}^{K-1}\left[\frac{\cos \phi_{k}}{d_{k}}-\frac{\cos \phi_{r}}{d_{r}}\right]\left[\frac{\sin \phi_{k}}{d_{k}}-\frac{\sin \phi_{r}}{d_{r}}\right] . \\
J_{y y}(\theta) & =\rho \sum_{k=1}^{K-1}\left[\frac{\sin \phi_{k}}{d_{k}}-\frac{\sin \phi_{r}}{d_{r}}\right]^{2} .
\end{aligned}
$$

Here, $\phi_{k} \in[0,2 \pi)$ is the angle the MN makes with respect to the $k^{\text {th }} \mathrm{AP}$ as illustrated in Fig. 1 , and $\rho=\left(\frac{10 \beta}{\tilde{\sigma} \ln 10}\right)^{2}$. If $\operatorname{var}(\widehat{\theta})_{K}$ denotes the variance of our location estimate, then from the CRLB property in (6), we have,

$$
\operatorname{var}(\widehat{\theta})_{K} \geq \frac{\lambda_{K}}{\rho \cdot \eta_{K}}
$$

where $\lambda_{K}=\sum_{k=1}^{K-1}\left(u_{k}^{2}+v_{k}^{2}\right), \eta_{K}=\sum_{k=1}^{K-1} u_{k}^{2} \sum_{k=1}^{K-1} v_{k}^{2}-$ $\left\{\sum_{k=1}^{K-1} u_{k} v_{k}\right\}^{2}, u_{k}=\left[\frac{\cos \phi_{k}}{d_{k}}-\frac{\cos \phi_{r}}{d_{r}}\right]$ and $v_{k}=\left[\frac{\sin \phi_{k}}{d_{k}}-\right.$ $\left.\frac{\sin \phi_{r}}{d_{r}}\right]$. The RHS of (9) specifies the CRLB of the MN's location estimate using SSD for a system having $K$ APs, i.e.,

$$
\mathcal{C}_{K}=\frac{\lambda_{K}}{\rho \cdot \eta_{K}} .
$$

The detailed calculation is presented in Appendix A. From (10), it is evident that the CRLB depends on i) the number of APs, $K$, ii) the geometry of the AP, $\phi_{k}$, iii) propagation model parameters, $\widetilde{\sigma}$ and $\beta$, and iv) the distance of the AP from the MN, $d_{k}$. Next, we elaborately discuss the properties of these parameters on location estimation error bound. Using the findings, we provide insights into positioning system deployment issues, and also propose a modified $\mathcal{K}$-NN scheme that shows improvement over the $\mathcal{K}$-NN algorithm.

\section{A. Impact of the Number of APs}

Theorem 1: The introduction of an additional AP with parameters $\left(d_{K+1}, \phi_{K+1}\right)$ results in the reduction of the CRLB except when $\phi_{K+1}=\phi_{r}=\phi_{k}, \forall k \in\{1,2, \ldots, K\}-\{r\}$.

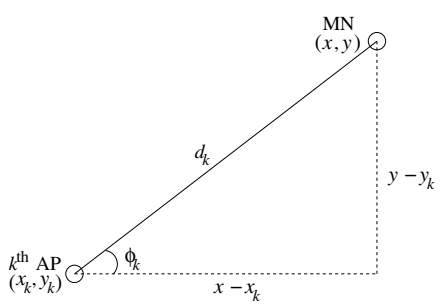

Fig. 1. Definition of angle $\phi_{k}$.

Proof: When an additional $(K+1)^{\text {th }}$ AP is added to the system of $K$ APs, the CRLB can be represented as,

$$
\mathcal{C}_{K+1}=\frac{\lambda_{K+1}}{\rho \cdot \eta_{K+1}}
$$

where $\lambda_{K+1}=\lambda_{K}+\left(u_{K+1}^{2}+v_{K+1}^{2}\right)$ and $\eta_{K+1}=$ $\eta_{K}+\left(v_{K+1}^{2} \sum_{k=1}^{K-1} u_{k}^{2}-2 \cdot u_{K+1} \cdot v_{K+1} \cdot \sum_{k=1}^{K-1} u_{k} v_{k}+\right.$ $u_{K+1}^{2} \sum_{k=1}^{K-1} v_{k}^{2}$ ). Our goal is to prove $\mathcal{C}_{K}-\mathcal{C}_{K+1} \geq 0$. Subtracting (11) from (10), we obtain,

$$
\begin{aligned}
\mathcal{C}_{K}-\mathcal{C}_{K+1}= & \left\{v_{K+1}^{2}\left(\lambda_{K} \sum_{k=1}^{K-1} u_{k}^{2}-\eta_{K}\right)+u_{K+1}^{2} .\right. \\
& \left(\lambda_{K} \sum_{k=1}^{K-1} v_{k}^{2}-\eta_{K}\right)-2 u_{K+1} \cdot v_{K+1} . \\
& \left.\lambda_{K} \sum_{k=1}^{K-1} u_{k} v_{k}\right\} /\left(\rho \cdot \eta_{K} \cdot \eta_{K+1}\right) .
\end{aligned}
$$

The denominator of (12) is non-negative since $\eta_{K}, \eta_{K+1} \geq 0$ (using the inequality $\sum_{k=1}^{K} u_{k}^{2} \sum_{k=1}^{K} v_{k}^{2}-\left\{\sum_{k=1}^{K} u_{k} v_{k}\right\}^{2} \geq$ 0 ; the induction proof of this inequality is omitted for brevity) and $\rho>0$ (see (25)). To prove the nonnegativity of the numerator of (12), we use the inequality, $\sqrt{\left(\lambda_{K} \sum_{k=1}^{K-1} u_{k}^{2}-\eta_{K}\right)\left(\lambda_{K} \sum_{k=1}^{K-1} v_{k}^{2}-\eta_{K}\right)} \geq$ $\lambda_{K} \sum_{k=1}^{K-1} u_{k} v_{k}$ (see Appendix B for proof) into (12),

$$
\begin{aligned}
& \geq\left\{v_{K+1}^{2}\left(\lambda_{K} \sum_{k=1}^{K-1} u_{k}^{2}-\eta_{K}\right)+u_{K+1}^{2} \cdot\right. \\
&\left(\lambda_{K} \sum_{k=1}^{K-1} v_{k}^{2}-\eta_{K}\right)-2 \cdot u_{K+1} \cdot v_{K+1} \cdot \\
&\left.\sqrt{\left(\lambda_{K} \sum_{k=1}^{K-1} u_{k}^{2}-\eta_{K}\right)\left(\lambda_{K} \sum_{k=1}^{K-1} v_{k}^{2}-\eta_{K}\right)}\right\} \\
& /\left(\rho \cdot \eta_{K} \cdot \eta_{K+1}\right) \\
&=\left\{v_{K+1} \sqrt{\left(\lambda_{K} \sum_{k=1}^{K-1} u_{k}^{2}-\eta_{K}\right)}-u_{K+1} \cdot\right. \\
& \geq\left.\sqrt{\left(\lambda_{K} \sum_{k=1}^{K-1} v_{k}^{2}-\eta_{K}\right)}\right\}^{2} /\left(\rho \cdot \eta_{K} \cdot \eta_{K+1}\right) \\
& 0 .
\end{aligned}
$$




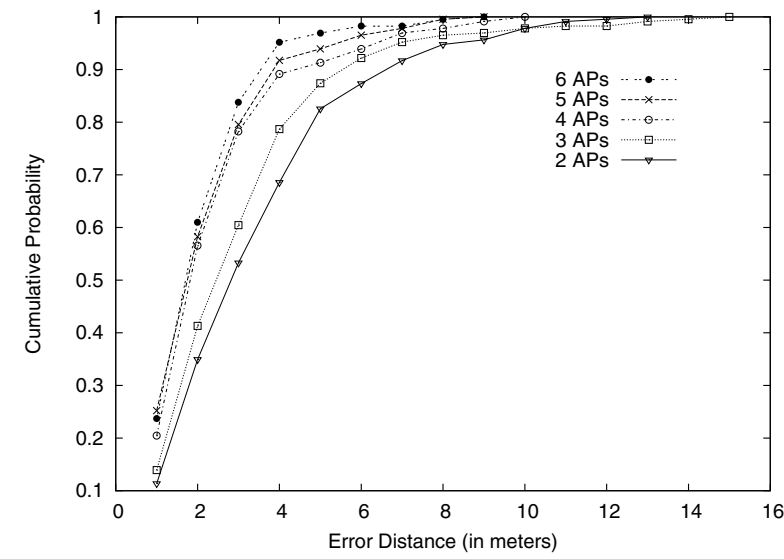

Fig. 2. Localization accuracy improves with increasing number of APs.

The case $\mathcal{C}_{K}-\mathcal{C}_{K+1}=0$ arises only when both the equality conditions of (27) and (13) hold. These two equalities are satisfied only for the scenario, $\phi_{K+1}=\phi_{r}=\phi_{k}, \forall k \in$ $\{1,2, \ldots, K\}-\{r\}$, i.e., when all the APs are collinear (see Appendix $\mathrm{C}$ for detailed calculation). Therefore, except for this situation, the introduction of an additional AP indeed lowers the CRLB of the location estimate.

Fig. 2 shows the experimental results (conducted in our testbed of Fig. 3) of localization accuracy as we vary the number of APs. It shows monotonic increase in localization accuracy as the number of APs increases. This experimental result is completely in sync with the findings of our CRLB analysis. We have used $\mathcal{K}$-NN algorithm discussed in Section III-B as our location classifier. However, from the pattern recognition theory [18], it is well-known that the dimension of the feature vector (i.e., number of APs in our case) cannot be arbitrarily increased to achieve better accuracy. As a matter of fact, we have not seen any improvement in localization accuracy when the number of APs is greater than ten.

\section{B. Impact of the Geometry of APs}

When $\phi_{k}=\phi, \forall k \in\{1,2, \ldots, K\}$, we see that the FIM (8) is singular. In other words, if all the APs lie on a straight line w.r.t. the $\mathrm{MN}$, then the location estimation error bound (10) is the largest.

Optimal geometry occurs when the MN is situated at the center of a $K$-sided regular polygon where the vertices of the polygon indicate the positions of the $K$ APs. In this setting, we have, $d_{k}=d, \forall k=\{1,2, \ldots, K\}$, and $\sum_{k=1}^{K} \sin \left(h \phi_{k}\right)=$ $\sum_{k=1}^{K} \cos \left(h \phi_{k}\right)=0$, for any integer, $h \geq 1$. Let us assume $\phi_{r}=0^{\circ}$, then the FIM (8) takes the following form,

$$
J(\theta)=\frac{\rho}{d^{2}}\left[\begin{array}{cc}
\frac{3 K}{2} & 0 \\
0 & \frac{K}{2}
\end{array}\right] .
$$

Using the terms of (14), we obtain the lower bound of $\operatorname{var}(\widehat{\theta})_{K}$ as $\frac{8 d^{2}}{3 \rho K}$, which suggests that it decreases with increasing $K$. In other words, the lower bound for a square configuration of the APs $(K=4)$ would be smaller than that of an equilateral triangle configuration $(K=3)$. Moreover, the lower bound may also decrease for the same number of APs' setup when they are closer to the $\mathrm{MN}$ (i.e., $d$ decreases) compared to the scenario when they are farther.

In Table I, we list the average localization errors when the equilateral triangle configuration of the three APs is considered for four different testing sets (the circular regions in Fig. 3). The testing set near the centroid of the equilateral triangle (Set 1 in Fig. 3) performs the best, which complies with the analysis here. Therefore, the system administrator may place the APs in such a way that a subset of them creates regular polygon with "popular area" being the centroid of it. Here, we have investigated the optimal APs' geometry from the CRLB perspective using SSD as location fingerprint. Experimental finding on optimal placement of APs for localization with traditional RSS fingerprint can be found in [19], [20] which shows similar trend.

Table II shows the average localization errors for the optimal and worst-case (collinear) configurations of the APs for a particular testing set (Set 1 of Fig. 3), which also supports our analysis. However, the collinear configuration of the APs is found to be better for signal coverage [20]. Since providing both data communication and location service could be the objectives of a Wi-Fi infrastructure, the system administrator needs to take these conflicting requirements into account during deployment.

\section{Impact of the Propagation Model Parameters}

From (10) and (25), it is evident that, if the shadowing variance of SSD, $\widetilde{\sigma}^{2}$ increases, then CRLB also increases. A lower value of path-loss exponent $\beta$ increases the CRLB as well. As a result, the location estimation error bound (10) would be larger in both cases. On the contrary, when the RSS shows less fluctuations (i.e., $\widetilde{\sigma} \downarrow$ ), the location fingerprints tend to be more consistent and are likely to produce better accuracy. The path-loss exponent $\beta$ is generally larger inside buildings which leads to lower CRLB in indoor environments compared to outdoors. Similar effects of these parameters are observed on accuracy for RSS-based localization as well [3], [15].

\section{Impact of the Distance of an AP from the MN}

In this section, we first analyze the impact of APs' distances on localization accuracy from the CRLB perspective. Subsequently, we utilize the finding of this analysis to define weights for the weighted least squares (WLS) approach which is shown to improve localization accuracy both analytically and experimentally. Finally, we take all these findings into account in order to modify our $\mathcal{K}$-NN algorithm in Section III-B that reduces the localization error further.

Under optimal geometric configuration of the APs, it has been seen in Section IV-B that, the setup with APs closer to the MN yields a reduction in the CRLB compared to the setting where the APs are farther. Now, let us investigate the effect of the distance of the $\mathrm{MN}$ from an $\mathrm{AP}$ in a more generic scenario.

Theorem 2: The decrease in any of the distances $d_{k}$ 's of the APs from the MN results in reduction of the CRLB. 


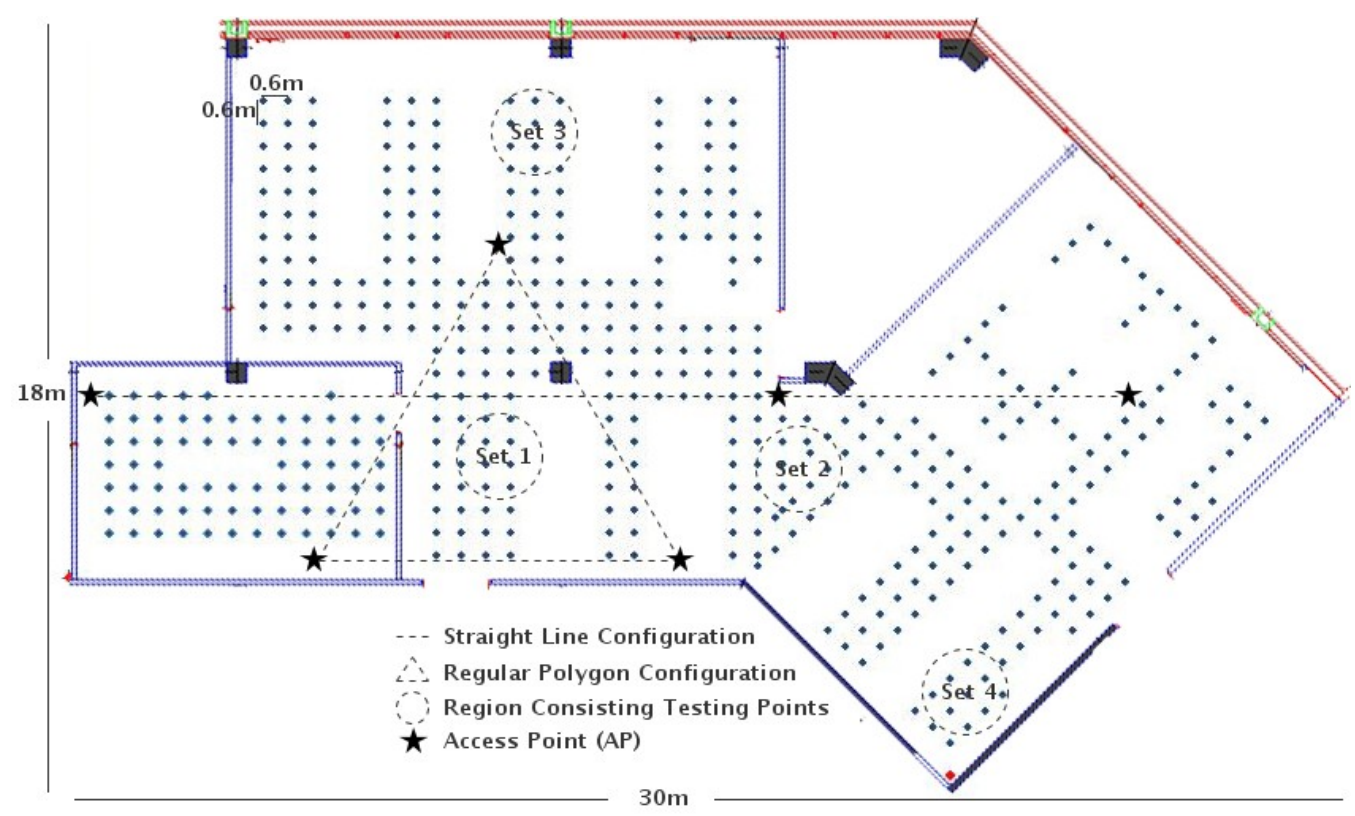

Fig. 3. Two different configurations of three APs: i) Regular Polygon and ii) Straight Line. The four testing sets are indicated by the circular regions.

TABLE I

AVERAGE LOCALIZATION ERRORS WHEN DIFFERENT TESTING SETS ARE USED FOR OPTIMAL CONFIGURATION OF THE THREE APS (THE EQUILATERAL TRIANGLE IN FIG. 3)

\begin{tabular}{c|c|c|c|c}
\hline Testing Points' Set & Set 1 & Set 2 & Set 3 & Set 4 \\
\hline Average Error (in meters) & 2.95 & 3.31 & 3.33 & 3.71 \\
\hline
\end{tabular}

TABLE II

AVERAGE LOCALIZATION ERRORS WHEN THE OPTIMAL (REGULAR POLYGON) AND WORST-CASE (STRAIGHT LINE) CONFIGURATIONS OF THE THREE APS ARE USED

\begin{tabular}{cc}
\hline Configuration & Average Error (in meters) \\
\hline Regular Polygon & 2.95 \\
Straight Line & 4.02 \\
\hline
\end{tabular}

Specifically, if $d_{k}^{\prime}=\alpha d_{k}, 0<\alpha \leq 1$, the new CRLB satisfies,

$$
\mathcal{C}_{K^{\prime}} \leq \mathcal{C}_{K}
$$

Proof: Suppose the reference $r^{\text {th }}$ AP is the farthest from the $\mathrm{MN}$, and for the ease of our calculation, we assume $d_{r} \rightarrow \infty$. From (10), we have, $\mathcal{C}_{K}=\frac{\lambda_{K}}{\rho \cdot \eta_{K}}$, where $\lambda_{K}=$ $\lambda_{K-1}+\frac{1}{d_{K}^{2}}$ and $\eta_{K}=\eta_{K-1}+v_{K}^{2} \sum_{k=1}^{K-2} u_{k}^{2}+u_{K}^{2} \sum_{k=1}^{K-2} v_{k}^{2}+$ $2 \cdot u_{K} \cdot v_{K} \cdot\left\{\sum_{k=1}^{K-2} u_{k} v_{k}\right\}$. Because of our assumption, the identities $u_{k}$ and $v_{k}$ take the following forms, $u_{k}=\frac{\cos \phi_{k}}{d_{k}}$ and $v_{k}=\frac{\sin \phi_{k}}{d_{k}}$. Similarly, the new CRLB can be written as, $\mathcal{C}_{K^{\prime}}=\frac{\lambda_{K^{\prime}}}{\rho \cdot \eta_{K^{\prime}}}$, where $\lambda_{K^{\prime}}=\lambda_{K-1}+\frac{1}{\alpha^{2} d_{K}^{2}}$ and $\eta_{K^{\prime}}=\eta_{K-1}+$ $v_{K^{\prime}}^{2} \sum_{k=1}^{K-2} u_{k}^{2}+u_{K^{\prime}}^{2} \sum_{k=1}^{K-2} v_{k}^{2}+2 \cdot u_{K^{\prime}} \cdot v_{K^{\prime}} \cdot\left\{\sum_{k=1}^{K-2} u_{k} v_{k}\right\}$ and the identities, $u_{K^{\prime}}=\frac{\cos \phi_{K}}{\alpha \cdot d_{K}}$ and $v_{K^{\prime}}=\frac{\sin \phi_{K}}{\alpha \cdot d_{K}}$.

Our goal is to prove $\mathcal{C}_{K}-\mathcal{C}_{K^{\prime}} \geq 0$. Using the CRLB expressions, we have,

$$
\mathcal{C}_{K}-\mathcal{C}_{K^{\prime}}=\frac{\lambda_{K} \eta_{K^{\prime}}-\lambda_{K^{\prime}} \eta_{K}}{\rho \cdot \eta_{K} \cdot \eta_{K^{\prime}}}
$$

The denominator of (16), $\rho \cdot \eta_{K} \cdot \eta_{K^{\prime}} \geq 0$. Now, we have to prove that the numerator of (16) is non-negative. The numerator can be simplified as,

$$
\begin{aligned}
\lambda_{K} \eta_{K^{\prime}}-\lambda_{K^{\prime}} \eta_{K} & \left(v_{K^{\prime}}^{2}-v_{K}^{2}\right)\left(\lambda_{K-1} \sum_{k=1}^{K-2} u_{k}^{2}-\eta_{K-1}\right) \\
& +\left(u_{K^{\prime}}^{2}-u_{K}^{2}\right)\left(\lambda_{K-1} \sum_{k=1}^{K-2} v_{k}^{2}-\eta_{K-1}\right) \\
& -2 \lambda_{K-1}\left(u_{K^{\prime}} v_{K^{\prime}}-u_{K} v_{K}\right)\left\{\sum_{k=1}^{K-2} u_{k} v_{k}\right\} .
\end{aligned}
$$

Using the inequality,

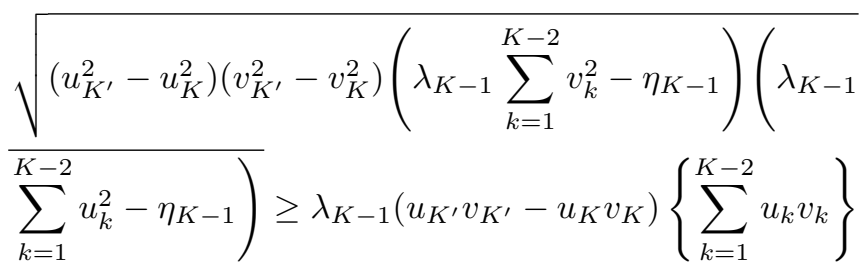

in (17) (the proof is shown in Appendix D), we obtain,

$$
\begin{aligned}
\mathcal{C}_{K}-\mathcal{C}_{K^{\prime}} \geq & \left\{\sqrt{\left(v_{K^{\prime}}^{2}-v_{K}^{2}\right)\left(\lambda_{K-1} \sum_{k=1}^{K-2} u_{k}^{2}-\eta_{K-1}\right)}\right. \\
& \left.-\sqrt{\left(u_{K^{\prime}}^{2}-u_{K}^{2}\right)\left(\lambda_{K-1} \sum_{k=1}^{K-2} v_{k}^{2}-\eta_{K-1}\right)}\right\}^{2} \\
\geq & \quad 0 .\left(\rho \cdot \eta_{K} \cdot \eta_{K^{\prime}}\right)
\end{aligned}
$$

The case $\mathcal{C}_{K}-\mathcal{C}_{K^{\prime}}=0$ arises when $\alpha=1$ (i.e., $d_{K^{\prime}}=d_{K}$ ) as the numerator (17) becomes zero. 
1) Weighted Least Squares (WLS) Approach: The CRLB given by (10) provides a benchmark for comparing the performance of location-estimation, but does not explicitly describe the estimator that achieves it [9]. In this section, using the impact of a closer AP in defining weights, we emerge with a WLS estimator that is shown to perform better than LS estimator both analytically and experimentally.

Let the coordinates of the $(K+1)$ APs, $\theta_{k}=\left[\begin{array}{ll}x_{k} & y_{k}\end{array}\right]^{T}$, $k=1,2, \ldots,(K+1)$ be known, and the MN's position is $\theta=\left[\begin{array}{ll}x & y\end{array}\right]^{T}$. The distances $d_{k}$ 's between the MN and the $K$ APs (excluding the reference $r^{\text {th }}$ AP) are calculated by means of linear RF propagation modeling using (1) as, $\left[\frac{p_{k}}{p_{r}}\right]_{\mathrm{dB}}=$ $a \log d_{k}+b$, where $a=-10 \beta$ and we assume $b=10 \beta \log d_{r}+$ $\left[X_{k}-X_{r}\right]_{\mathrm{dB}}$. Therefore, we have $K$ equations of the form, $\left\|\theta-\theta_{k}\right\|^{2}=\|\theta\|^{2}+\left\|\theta_{k}\right\|^{2}-2 \theta_{k}^{T} \theta=d_{k}^{2}, k=1,2, \ldots, K$.

Taking the difference of each of the above $K$ equations, this system transforms into a set of $\left(\begin{array}{c}K \\ 2\end{array}\right)$ linear equations,

$$
2\left(\theta_{k}-\theta_{l}\right)^{T} \theta=\left(\left\|\theta_{k}\right\|^{2}-\left\|\theta_{l}\right\|^{2}\right)-\left(d_{k}^{2}-d_{l}^{2}\right) .
$$

The above set of linear equations is of the form $y=\mathcal{X} \theta$, where $y$ is a column vector of length $\left(\begin{array}{c}K \\ 2\end{array}\right)$ whose components are from $\left(\left\|\theta_{k}\right\|^{2}-\left\|\theta_{l}\right\|^{2}\right)-\left(d_{k}^{2}-d_{l}^{2}\right)$, and $\mathcal{X}$ is a $\left(\begin{array}{c}K \\ 2\end{array}\right) \times 2$ matrix whose rows are from the set $\left\{2\left(\theta_{k}-\theta_{l}\right)^{T}\right\}, k=1,2 \ldots, K-1, l>$ $k$. The observations can be represented as, $y=\mathcal{X} \theta+\mathcal{N}$, where $\mathcal{N}$ is a zero-mean noise column vector of length $\left(\begin{array}{c}K \\ 2\end{array}\right)$. The general weighted quadratic risk function is of the form,

$$
R(\theta)_{\mathrm{WLS}}=\left[(y-\mathcal{X} \theta)^{T} \mathcal{W}^{T}(y-\mathcal{X} \theta)\right],
$$

where $\mathcal{W}$ is a $\left(\begin{array}{c}K \\ 2\end{array}\right) \times\left(\begin{array}{c}K \\ 2\end{array}\right)$ symmetric weight matrix. The WLS estimate of the node's location is given by [21],

$$
\widehat{\theta}=\left(\mathcal{X}^{T} \mathcal{W} \mathcal{X}\right)^{-1} \mathcal{X}^{T} \mathcal{W} y
$$

and the covariance matrix [21],

$$
\Psi_{\mathrm{WLS}}=\left(\mathcal{X}^{T} \mathcal{W} \mathcal{X}\right)^{-1} \mathcal{X}^{T} \mathcal{W} \Lambda \mathcal{W} \mathcal{X}\left(\mathcal{X}^{T} \mathcal{W} \mathcal{X}\right)^{-1}
$$

When the observation errors are uncorrelated, the weight matrix, $\mathcal{W}$, is diagonal. The resulting estimator is the best linear unbiased estimator (BLUE) if the weight matrix is equal to the inverse of the covariance matrix of the observation errors [22], i.e., $\mathcal{W}=\Lambda^{-1}$, where $\Lambda$ is the covariance matrix of the observation errors. Using this identity in (20), the best linear unbiased estimator's covariance matrix can be represented as, $\Psi_{\mathrm{BLUE}}=\left(\mathcal{X}^{T} \Lambda^{-1} \mathcal{X}\right)^{-1}$. Using matrix algebra, it can be shown that for any other choice of the weight matrix $\mathcal{W}$ [22], $\Psi_{\text {BLUE }} \leq \Psi_{\text {WLS }}$.

Let us consider the scenario of Fig. 4 where $\mathrm{AP}_{1}$ is first stationed far from the testing set. Suppose $\mathcal{W}$ is defined as the inverse of the covariance matrix of the observation errors:

$$
\mathcal{W}=\Lambda^{-1}=\left[\begin{array}{ccccc}
\frac{1}{\sigma_{1}^{2}+\sigma_{2}^{2}} & \cdots & 0 & \cdots & 0 \\
\vdots & \ddots & \vdots & & \vdots \\
0 & \cdots & \frac{1}{\sigma_{1}^{2}+\sigma_{K}^{2}} & \cdots & 0 \\
\vdots & & \vdots & \ddots & \vdots \\
0 & \cdots & 0 & \cdots & \frac{1}{\sigma_{K-1}^{2}+\sigma_{K}^{2}}
\end{array}\right]
$$

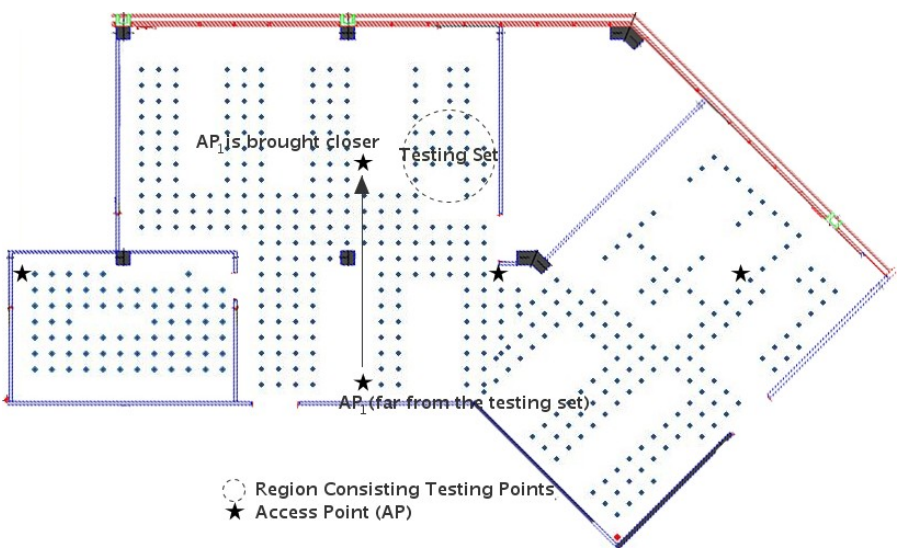

Fig. 4. From a distant position, $\mathrm{AP}_{1}$ is brought closer to the testing set which is indicated by the circular region. The other APs' positions are collinear.

Consequently, the matrix $\mathcal{X}^{T} \Lambda^{-1} \mathcal{X}$ can be evaluated as (22), shown at the top of the next page. Now, suppose $\mathrm{AP}_{1}$ is brought closer to the testing set (i.e., MN) as shown in Fig. 4. Let us denote this new distance as $d_{1}^{\prime}$ and the corresponding observation error variance and covariance matrix as ${\sigma_{1}^{\prime}}^{2}$ and $\Lambda^{\prime}$, respectively. $\mathcal{W}$ is expected to vary due to the observation in localization literature [1] that, the measurement errors associated with the closer $d_{1}^{\prime}$ calculation are generally less error-prone than that of the distant $d_{1}$ case, i.e., ${\sigma_{1}^{\prime}}^{2} \leq{\sigma_{1}}^{2}$. For ease of calculation, let us assume that the new (closer) position of $\mathrm{AP}_{1}$ and its old (farther) position are symmetric to each other with respect to the straight line formed by the other APs. Under this assumption, the new matrix $\mathcal{X}^{T} \Lambda^{\prime-1} \mathcal{X}$ takes the form of (23), shown at the top of the next page.

Using (22) and (23) and the identity ${\sigma_{1}^{\prime 2}}^{2} \leq \sigma_{1}^{2}$, it can be easily seen that, the matrix $\left(\mathcal{X}^{T} \Lambda^{\prime-1} \mathcal{X}-\mathcal{X}^{T} \Lambda^{-1} \mathcal{X}\right)$ is positive semidefinite. Therefore, we obtain, $\mathcal{X}^{T} \Lambda^{\prime-1} \mathcal{X} \geq \mathcal{X}^{T} \Lambda^{-1} \mathcal{X}$ which in turn yields $\left(\mathcal{X}^{T} \Lambda^{\prime-1} \mathcal{X}\right)^{-1} \leq\left(\mathcal{X}^{T} \Lambda^{-1} \mathcal{X}\right)^{-1}$, i.e., $\Psi_{\text {BLUE }}^{\prime} \leq \Psi_{\text {BLUE. }}$. In other words, considering an AP closer to the MN rather than a distant one produces a smaller covariance matrix, thereby, resulting in a better estimator. Fig. 5 shows our experimental results which strengthen this fact. We see that, the localization accuracy improves for both LS and WLS approaches when a near AP is considered in place of a distant one. Moreover, WLS's performance is better than the normal LS approach. We conclude this section with some remarks:

Remark 1: The least squares' (both LS and WLS) results in Fig. 5 are worse than the $\mathcal{K}$-NN. We attribute this inferior performance to the simple linear RF propagation model we have used in LS approaches to infer the distances $d_{k}$ 's.

Remark 2: The measurement errors in $\mathcal{W}(21)$ are calculated as deviations of the real and predicted distances between the MN and the APs. In doing so, we have assumed the real distances between the MN and the APs to be known which is usually not true in practice. Our modified $\mathcal{K}$-NN algorithm (described in the next section) does not suffer from this issue.

2) Modification to the $\mathcal{K}-N N$ Algorithm: It has been seen in Theorem 2 that the CRLB is reduced when we consider a closer AP compared to a distant one. We also applied this property to a well-known estimator algorithm (WLS) in the 


$$
\begin{aligned}
& \mathcal{X}^{T} \Lambda^{-1} \mathcal{X}=\left[\begin{array}{cc}
\sum_{\substack{k=1 \\
k<l \leq K}}^{K-1} \frac{\left(x_{k}-x_{l}\right)^{2}}{\sigma_{k}^{2}+\sigma_{l}^{2}} & \sum_{\substack{k=1 \\
k<l \leq K}}^{K-1} \frac{\left(x_{k}-x_{l}\right)\left(y_{k}-y_{l}\right)}{\sigma_{k}^{2}+\sigma_{l}^{2}} \\
\sum_{\substack{k=1 \\
k<l \leq K}} \frac{\left(x_{k}-x_{l}\right)\left(y_{k}-y_{l}\right)}{\sigma_{k}^{2}+\sigma_{l}^{2}} & \sum_{\substack{k=1 \\
k<l \leq K}}^{K-1} \frac{\left(y_{k}-y_{l}\right)^{2}}{\sigma_{k}^{2}+\sigma_{l}^{2}}
\end{array}\right] \\
& \mathcal{X}^{T} \Lambda^{\prime-1} \mathcal{X}=\left[\begin{array}{ccc}
\sum_{\substack{k=2 \\
k<=K}}^{K-1} \frac{\left(x_{k}-x_{l}\right)^{2}}{\sigma_{k}^{2}+\sigma_{l}^{2}}+\sum_{l=2}^{K} \frac{\left(x_{1}-x_{l}\right)^{2}}{\sigma_{1}^{\prime 2}+\sigma_{l}^{2}} & \sum_{\substack{k=2 \\
k<l \leq K}}^{K-1} \frac{\left(x_{k}-x_{l}\right)\left(y_{k}-y_{l}\right)}{\sigma_{k}^{2}+\sigma_{l}^{2}}+\sum_{l=2}^{K} \frac{\left(x_{1}^{\prime}-x_{l}\right)\left(y_{1}^{\prime}-y_{l}\right)}{\sigma_{1}^{\prime 2}+\sigma_{l}^{2}} \\
\sum_{\substack{k=2 \\
k<l \leq K}}^{K-1} \frac{\left(x_{k}-x_{l}\right)\left(y_{k}-y_{l}\right)}{\sigma_{k}^{2}+\sigma_{l}^{2}}+\sum_{l=2}^{K} \frac{\left(x_{1}^{\prime}-x_{l}\right)\left(y_{1}^{\prime}-y_{l}\right)}{\sigma_{1}^{\prime 2}+\sigma_{l}^{2}} & \sum_{\substack{k=2 \\
k<l \leq K}}^{K-1} \frac{\left(y_{k}-y_{l}\right)^{2}}{\sigma_{k}^{2}+\sigma_{l}^{2}}+\sum_{l=2}^{K} \frac{\left(y_{1}-y_{l}\right)^{2}}{\sigma_{1}^{\prime 2}+\sigma_{l}^{2}}
\end{array}\right]
\end{aligned}
$$

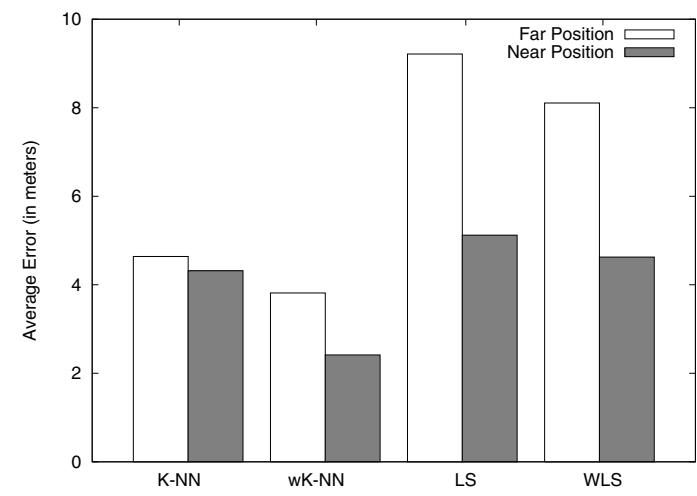

Fig. 5. Average localization errors of four different algorithms for two different placements of $\mathrm{AP}_{1}$ (near vs. far as shown in Fig. 4). The testing set is indicated by the circular region in Fig. 4.

previous section, and show that its accuracy improves, both analytically and experimentally.

Utilizing the above two observations, we modified our $\mathcal{K}$-NN algorithm presented in Section III-B into a weighted $\mathcal{K}$-NN scheme where the conditional probability is now changed to $\operatorname{Pr}\left(o_{j} \mid c_{i}\right)=$ $\prod_{k=1}^{K-1}\left\{\left[\frac{1}{d_{k i}^{\gamma}} \cdot \operatorname{Pr}\left(p_{k}-p_{r} \mid c_{i}\right)\right] /\left(\sum_{i=1}^{L} \frac{1}{d_{k i}^{\gamma}}\right)\right\}$. Here, $\quad L$ is the number of training locations and $\gamma(\gamma \geq 0)$ is the weight exponent. The weight is chosen in such a way that the closer APs are given more importance. We have chosen $\gamma$ empirically as 2 . Note that, $\gamma=0$ transforms our weighted $\mathcal{K}$-NN into the normal $\mathcal{K}$-NN algorithm. The rest of the algorithm is exactly similar to the one described in Section III-B.

Fig. 5 shows that the weighted $\mathcal{K}-\mathrm{NN}(\mathrm{w} \mathcal{K}-\mathrm{NN})$ algorithm's performance is better than the $\mathcal{K}$-NN (see the first two pairs of bars from the left). Moreover, the localization accuracy of the $\mathrm{w} \mathcal{K}-\mathrm{NN}$ is further improved when one of the APs $\left(\mathrm{AP}_{1}\right)$ can be placed closer to the testing set.

\section{CONCLUSION}

In this paper, we studied the CRLB of localization using SSD as location fingerprint. We investigated the effects of various parameters (e.g., quantity and geometry of the APs, the distances of the APs from the MN, etc.) of this bound on localization error. Subsequently, we utilize one of the findings of our analysis, which reveals that a closer AP to the MN is more important for localization purpose compared to a distant one, in order to define weights for a wK $\mathcal{K}-\mathrm{NN}$ algorithm. Moreover, we also provide design phase suggestions for various geometric configurations and quantities of the APs by revealing error trends associated with them. In short, our analysis and experimental results provide valuable insights into the localization performance and deployment issues of a positioning system based on SSD. We expect SSD to be the preferred choice of location fingerprint over RSS if a positioning system aims to provide services to devices with heterogeneous hardware solutions. Our analysis and findings in this paper should open the door for further analysis and designing of various efficient localization algorithms where SSD is chosen as the location fingerprint.

\section{ACKNOWLEDGMENTS}

This research work has been supported in part by National Research Foundation project grant NRF2007IDM-IDM002069 on "Life Spaces".

\section{APPENDIX A}

\section{DETAILED CALCULATION OF CRLB FOR LOCALIZATION USING SSD AS LOCATION FINGERPRINT}

The joint p.d.f. (7) of the $(K-1)$ independent SSD measurements can be simplified as,

$$
\begin{aligned}
f_{\theta}(P)=\prod_{k=1}^{K-1} \frac{1}{\sqrt{2 \pi} \widetilde{\sigma}} \frac{10}{\ln 10} \frac{p_{r}}{p_{k}} \exp \left\{-\frac{\rho}{8}\left(\ln \frac{d_{k}^{2}}{\tilde{d}_{k r}^{2}}\right)^{2}\right\}, \\
\text { where } \rho=\left(\frac{10 \beta}{\widetilde{\sigma} \ln 10}\right)^{2} \\
\text { and } \tilde{d}_{k r}=d_{r}\left(\frac{p_{r}}{p_{k}}\right)^{\frac{1}{\beta}} .
\end{aligned}
$$

Consequently, the log-likelihood of (24) takes the form, $\ln f_{\theta}(P)=\sum_{k=1}^{K-1}\left[C_{k r}-\frac{\rho}{8}\left(\ln \frac{d_{k}^{2}}{\tilde{d}_{k r}^{2}}\right)^{2}\right]$, where $C_{k r}=$ Constant w.r.t. $\theta=\frac{1}{\sqrt{2 \pi} \tilde{\sigma}} \frac{10}{\ln 10} \frac{p_{r}}{p_{k}}, \quad k=1,2, \ldots,(K-1)$. 
To derive the entries of (8), we calculate the score function,

$$
\begin{aligned}
U(\theta)= & \left\{\frac{\partial}{\partial x} \ln f_{\theta}(P), \frac{\partial}{\partial y} \ln f_{\theta}(P)\right\} \\
= & \left\{-\frac{\rho}{2} \sum_{k=1}^{K-1}\left(\ln \frac{d_{k}^{2}}{\tilde{d}_{k r}^{2}}\right)\left[\frac{\left(x-x_{k}\right)}{d_{k}^{2}}-\frac{\left(x-x_{r}\right)}{d_{r}^{2}}\right],\right. \\
& \left.-\frac{\rho}{2} \sum_{k=1}^{K-1}\left(\ln \frac{d_{k}^{2}}{\tilde{d}_{k r}^{2}}\right)\left[\frac{\left(y-y_{k}\right)}{d_{k}^{2}}-\frac{\left(y-y_{r}\right)}{d_{r}^{2}}\right]\right\} .
\end{aligned}
$$

Taking the derivative of $U(\theta)$ w.r.t. $\theta$, we obtain,

$$
\begin{aligned}
& \frac{\partial}{\partial x^{2}} \ln f_{\theta}(S)=-\frac{\rho}{2} \sum_{k=1}^{K-1}\left\{\left(\ln \frac{d_{k}^{2}}{\tilde{d}_{k r}^{2}}\right) \times\right. \\
& \left.\frac{\partial}{\partial x}\left[\frac{\left(x-x_{k}\right)}{d_{k}^{2}}-\frac{\left(x-x_{r}\right)}{d_{r}^{2}}\right]+2\left[\frac{\left(x-x_{k}\right)}{d_{k}^{2}}-\frac{\left(x-x_{r}\right)}{d_{r}^{2}}\right]^{2}\right\} . \\
& \frac{\partial}{\partial y \partial x} \ln f_{\theta}(S)=\frac{\partial}{\partial x \partial y} \ln f_{\theta}(S) \\
& =-\frac{\rho}{2} \sum_{k=1}^{K-1}\left\{\left(\ln \frac{\left.d_{k}^{2}\right)}{\tilde{d}_{k r}^{2}}\right) \frac{\partial}{\partial y}\left[\frac{\left(x-x_{k}\right)}{d_{k}^{2}}-\frac{\left(x-x_{r}\right)}{d_{r}^{2}}\right]\right. \\
& \left.+2\left[\frac{\left(x-x_{k}\right)}{d_{k}^{2}}-\frac{\left(x-x_{r}\right)}{d_{r}^{2}}\right]\left[\frac{\left(y-y_{k}\right)}{d_{k}^{2}}-\frac{\left(y-y_{r}\right)}{d_{r}^{2}}\right]\right\} . \\
& \frac{\partial}{\partial y^{2}} \ln f_{\theta}(S)=-\frac{\rho}{2} \sum_{k=1}^{K-1}\left\{\left(\ln \frac{\left.d_{k}^{2}\right) \times \tilde{d}_{k r}^{2}}{f^{2}}\right)\right. \\
& \left.\frac{\partial}{\partial y}\left[\frac{\left(y-y_{k}\right)}{d_{k}^{2}}-\frac{\left(y-y_{r}\right)}{d_{r}^{2}}\right]+2\left[\frac{\left(y-y_{k}\right)}{d_{k}^{2}}-\frac{\left(y-y_{r}\right)}{d_{r}^{2}}\right]^{2}\right\} .
\end{aligned}
$$

We see that, all the elements of derivatives of the score function depend on a term, $\left(\ln \frac{d_{k}^{2}}{\tilde{d}_{k r}^{2}}\right)$, which has an expected value of zero. Therefore, the entries of (8) become,

$$
\begin{aligned}
J_{x x}(\theta) & =\rho \sum_{k=1}^{K-1}\left[\frac{\left(x-x_{k}\right)}{d_{k}^{2}}-\frac{\left(x-x_{r}\right)}{d_{r}^{2}}\right]^{2} \\
& =\rho \sum_{k=1}^{K-1}\left[\frac{\cos \phi_{k}}{d_{k}}-\frac{\cos \phi_{r}}{d_{r}}\right]^{2} \cdot \\
J_{x y}(\theta) & =J_{y x}(\theta) \\
& =\rho \sum_{k=1}^{K-1}\left\{\left[\frac{\left(x-x_{k}\right)}{d_{k}^{2}}-\frac{\left(x-x_{r}\right)}{d_{r}^{2}}\right] \times\right. \\
& =\rho \sum_{k=1}^{K-1}\left[\frac{\left(y-y_{k}\right)}{\left.\left.d_{k}^{2}-\frac{\left(y-y_{r}\right)}{d_{r}^{2}}\right]\right\}}\right. \\
J_{y y}(\theta) & \rho \sum_{k=1}^{K-1}\left[\frac{\left(x-x_{k}\right)}{d_{k}^{2}}-\frac{\left(x-x_{r}\right)}{d_{r}^{2}}\right]^{2} \\
& \left.\rho \sum_{k=1}^{K-1}\left[\frac{\sin \phi_{k}}{d_{k}}-\frac{\sin \phi_{r}}{d_{r}}\right]_{k}^{2} \cdot \frac{\sin \phi_{r}}{d_{r}}\right] .
\end{aligned}
$$

Here, $\phi_{k} \in[0,2 \pi)$ is the angle the $\mathrm{MN}$ makes w.r.t. the $k^{\text {th }}$ AP as illustrated in Fig. 1. Subsequently, the CRLB can be expressed as,

$$
\begin{aligned}
& \{J(\theta)\}^{-1}=\frac{1}{|J(\theta)|}\left[\begin{array}{cc}
J_{y y}(\theta) & -J_{x y}(\theta) \\
-J_{y x}(\theta) & J_{x x}(\theta)
\end{array}\right], \\
& \text { where }|J(\theta)|=J_{x x}(\theta) \cdot J_{y y}(\theta)-J_{x y}(\theta) \cdot J_{y x}(\theta) .
\end{aligned}
$$

Suppose the variance of the location estimate of SSD-based localization with $K$ APs is denoted as $\operatorname{var}(\widehat{\theta})_{K}$. From the CRLB property (6), we know that, $\operatorname{Cov}(\widehat{\theta}, \theta) \geq\{J(\theta)\}^{-1}$, i.e., the matrix $\operatorname{Cov}(\widehat{\theta}, \theta)-\{J(\theta)\}^{-1}$ is positive semidefinite [9]. Since the diagonal elements of positive semidefinite matrices are larger or equal to zero, we obtain the following inequalities for any unbiased estimator using the identities of (3) and (25),

$$
\sigma_{\widehat{x}}^{2} \geq \frac{J_{y y}(\theta)}{|J(\theta)|} \quad \text { and } \quad \sigma_{\widehat{y}}^{2} \geq \frac{J_{x x}(\theta)}{|J(\theta)|} .
$$

Consequently, we have,

$$
\begin{aligned}
\operatorname{var}(\widehat{\theta})_{K}=\sigma_{\widehat{x}}^{2}+\sigma_{\widehat{y}}^{2} & \geq \frac{J_{x x}(\theta)+J_{y y}(\theta)}{|J(\theta)|} \\
& =\frac{\lambda_{K}}{\rho \cdot \eta_{K}},
\end{aligned}
$$

where we define, $\lambda_{K}=\sum_{k=1}^{K-1}\left(u_{k}^{2}+v_{k}^{2}\right), \quad \eta_{K}=$ $\sum_{k=1}^{K-1} u_{k}^{2} \sum_{k=1}^{K-1} v_{k}^{2}-\left\{\sum_{k=1}^{K-1} u_{k} v_{k}\right\}^{2}, u_{k}=\left[\frac{\cos \phi_{k}}{d_{k}}-\right.$ $\left.\frac{\cos \phi_{r}}{d_{r}}\right]$ and $v_{k}=\left[\frac{\sin \phi_{k}}{d_{k}}-\frac{\sin \phi_{r}}{d_{r}}\right]$.

$$
\begin{gathered}
\text { APPENDIX B } \\
\text { Proof of } \\
\sqrt{\left(\lambda_{K} \sum_{k=1}^{K-1} u_{k}^{2}-\eta_{K}\right)\left(\lambda_{K} \sum_{k=1}^{K-1} v_{k}^{2}-\eta_{K}\right)} \geq \\
\lambda_{K} \sum_{k=1}^{K-1} u_{k} v_{k} .
\end{gathered}
$$

Simplifying the LHS of the inequality, we obtain,

$$
\begin{aligned}
& \sqrt{\left(\lambda_{K} \sum_{k=1}^{K-1} u_{k}^{2}-\eta_{K}\right)\left(\lambda_{K} \sum_{k=1}^{K-1} v_{k}^{2}-\eta_{K}\right)} \\
= & \sqrt{\lambda_{K}^{2} \sum_{k=1}^{K-1} u_{k}^{2} \sum_{k=1}^{K-1} v_{k}^{2}-\eta_{K} \cdot \lambda_{K} \sum_{k=1}^{K-1}\left(u_{k}^{2}+v_{k}^{2}\right)+\eta_{K}^{2}} \\
= & \sqrt{\lambda_{K}^{2} \cdot \eta_{K}+\lambda_{K}^{2}\left\{\sum_{k=1}^{K-1} u_{k} v_{k}\right\}^{2}-\eta_{K} \cdot \lambda_{K}^{2}+\eta_{K}^{2}} \\
= & \sqrt{\lambda_{K}^{2}\left\{\sum_{k=1}^{K-1} u_{k} v_{k}\right\}+\eta_{K}^{2}} \\
\geq & \lambda_{K} \sum_{k=1}^{K-1} u_{k} v_{k}, \quad \text { since } \eta_{K}^{2} \geq 0 .
\end{aligned}
$$

\section{APPENDIX C}

PROOF OF $\phi_{K+1}=\phi_{r}=\phi_{k}, \forall k \in\{1,2, \ldots, K\}-\{r\}$

$$
\text { WHEN } \mathcal{C}_{K}-\mathcal{C}_{K+1}=0
$$

Here, we give the proof of the claim that, the equality conditions of both (27) and (13) result in the following, $\phi_{K+1}=\phi_{r}=\phi_{k}, \forall k \in\{1,2, \ldots, K\}-\{r\}$. 
The equality condition of (27) requires, $\eta_{K}^{2}=0$, i.e., $\eta_{K}=$ 0 . Consequently, from the definition of $\eta_{K}$, we can write,

$$
\sum_{k=1}^{K-1} u_{k}^{2} \sum_{k=1}^{K-1} v_{k}^{2}=\left\{\sum_{k=1}^{K-1} u_{k} v_{k}\right\}^{2}
$$

Using the identities of $u_{k}$ and $v_{k}$, it can be deduced that, only when $\phi_{k}=\phi_{r}, \forall k \in\{1,2, \ldots, K\}-\{r\}$, the LHS and RHS of (28) become equal.

Now, putting $\eta_{K}=0$ into the equality condition of (13), we obtain,

$$
v_{K+1}^{2} \sum_{k=1}^{K-1} u_{k}^{2}=u_{K+1}^{2} \sum_{k=1}^{K-1} v_{k}^{2} .
$$

Plugging the values of $u_{\mathrm{K}+1}, v_{\mathrm{K}+1}, u_{k}$ and $v_{k}$ into (29), we get,

$$
\begin{aligned}
& {\left[\frac{\sin \phi_{K+1}}{d_{K+1}}-\frac{\sin \phi_{r}}{d_{r}}\right]^{2} \sum_{k=1}^{K-1}\left[\frac{\cos \phi_{k}}{d_{k}}-\frac{\cos \phi_{r}}{d_{r}}\right]^{2} } \\
= & {\left[\frac{\cos \phi_{K+1}}{d_{K+1}}-\frac{\cos \phi_{r}}{d_{r}}\right]^{2} \sum_{k=1}^{K-1}\left[\frac{\sin \phi_{k}}{d_{k}}-\frac{\sin \phi_{r}}{d_{r}}\right]^{2} . }
\end{aligned}
$$

Putting $\phi_{k}=\phi_{r}, \forall k \in\{1,2, \ldots, K\}-\{r\}$ (derived from the equality condition of (27) above) into (30), we have,

$$
\begin{aligned}
& \cos ^{2} \phi_{r}\left[\frac{\sin \phi_{K+1}}{d_{K+1}}-\frac{\sin \phi_{r}}{d_{r}}\right]^{2} \\
= & \sin ^{2} \phi_{r}\left[\frac{\cos \phi_{K+1}}{d_{K+1}}-\frac{\cos \phi_{r}}{d_{r}}\right]^{2} .
\end{aligned}
$$

Simplifying (31), it can be easily seen that, $\phi_{K+1}=\phi_{r}$. Combining this result with $\phi_{k}=\phi_{r}, \forall k \in\{1,2, \ldots, K\}-\{r\}$, we finally obtain, $\phi_{K+1}=\phi_{r}=\phi_{k}, \forall k \in\{1,2, \ldots, K\}-\{r\}$.

\section{APPENDIX D}

\section{PROOF OF INEQUALITY}

$\frac{\sqrt{\left(u_{K^{\prime}}^{2}-u_{K}^{2}\right)\left(v_{K^{\prime}}^{2}-v_{K}^{2}\right)\left(\lambda_{K-1} \sum_{k=1}^{K-2} v_{k}^{2}-\eta_{K-1}\right)\left(\lambda_{K-1}\right.}}{\left.\sum_{k=1}^{K-2} u_{k}^{2}-\eta_{K-1}\right)} \geq \lambda_{K-1}\left(u_{K^{\prime}} v_{K^{\prime}}-u_{K} v_{K}\right) \sum_{k=1}^{K-2} u_{k} v_{k}$.

Simplifying the LHS of the above inequality we get,

$$
\begin{aligned}
& \frac{\left(u_{K^{\prime}} v_{K^{\prime}}-u_{K} v_{K}\right) \sqrt{\lambda_{K-1}^{2} \sum_{k=1}^{K-2} u_{k}^{2} \sum_{k=1}^{K-2} v_{k}^{2}-\eta_{K-1}}}{\lambda_{K-1}\left\{\sum_{k=1}^{K-2}\left(u_{k}^{2}+v_{k}^{2}\right)\right\}+\eta_{K-1}^{2}} \\
= & \left(u_{K^{\prime}} v_{K^{\prime}}-u_{K} v_{K}\right) \sqrt{\lambda_{K-1}^{2}\left[\eta_{K-1}+\left\{\sum_{k=1}^{K-2} u_{k} v_{k}\right\}^{2}\right]-} \\
& \frac{\eta_{K-1} \cdot \lambda_{K-1}^{2}+\eta_{K-1}^{2}}{\geq} \lambda_{K-1}\left(u_{K^{\prime}} v_{K^{\prime}}-u_{K} v_{K}\right)\left\{\sum_{k=1}^{K-2} u_{k} v_{k}\right\}, \text { since } \eta_{K-1}^{2} \geq 0 .
\end{aligned}
$$

\section{REFERENCES}

[1] P. Bahl and V. N. Padmanabhan, "RADAR: An in-building RF-based user location and tracking system," in Proc. IEEE INFOCOM, Tel Aviv, Israel, Mar. 2000, pp. 775-784.

[2] P. Castro, P. Chiu, T. Kremenek, and R. R. Muntz, "A probabilistic room location service for wireless networked environments," in Proc. UbiComp'01, Atlanta, GA, Sept. 2001, pp. 18-34.

[3] K. Kaemarungsi and P. Krishnamurthy, "Modeling of indoor positioning systems based on location fingerprinting," in Proc. IEEE INFOCOM'04, Mar. 2004, pp. 1012-1022.

[4] M. A. Youssef, A. Agrawala, and A. U. Shankar, "WLAN location determination via clustering and probability distributions," in Proc. IEEE PERCOM'03, Mar. 2003.

[5] P. Tao, A. Rudys, A. M. Ladd, and D. S. Wallach, "Wireless LAN location-sensing for security applications," in Proc. ACM WiSe'03, San Diego, CA, Sept. 2003, pp. 11-20.

[6] M. Hossain, H. Nguyen Van, Y. Jin and W.-S. Soh, "Indoor localization using multiple wireless technologies," in Proc. IEEE MASS, Pisa, Italy, Oct. 2007. [Online]. Available: http://www.ece.nus.edu.sg/stfpage/ elesohws/mass07.pdf

[7] A. Haeberlen, E. Flannery, A. M. Ladd, A. Rudys, D. S. Wallach, and L. E. Kavraki, "Practical robust localization over large-scale 802.11 wireless networks," in Proc. ACM MobiCom'04, PA, 2004, pp. 70-84.

[8] M. B. Kjaergaard and C. V. Munk, "A calibration-free solution for handling differences in signal strength," in Proc. IEEE PerCom'08, Mar. 2008.

[9] H. L. V. Trees, Optimum array processing: Part IV of detection, estimation and modulation theory. John Wiley \& Sons, Inc., 2002.

[10] Y. Qi, H. Suda, and H. Kobayashi, "On time-of-arrival positioning in a multipath environment," in Proc. IEEE VTC'04, Sept. 2004.

[11] A. Dersan and Y. Tanik, "Passive radar localization by time difference of arrival," in Proc. MILCOM, Oct. 2002, pp. 1251-1257.

[12] C. Chang and A. Sahai, "Estimation bounds for localization," in Proc IEEE SECON'04, Oct. 2004, pp. 415-424

[13] S. Venkatesh and R. M. Buehrer, "Multiple-access insights from bounds on sensor localization," Pervasive and Mobile Computing, vol. 4, no. 1, pp. 33-61, 2008.

[14] N. Patwari and A. O. Hero, "Location estimation accuracy in wireless sensor networks," in Proc. IEEE Asilomar Conference on Signals and Systems, Pacific Grove, CA, Nov. 2002, pp. 1523-1527.

[15] K. Yedavalli, B. Krishnamachari, S. Ravula, and B. Srinivasan, "Ecolocation: a sequence based technique for rf localization in wireless sensor networks," in Proc. ISPN'05, Apr. 2005.

[16] K. Kaemarungsi and P. Krishnamurthy, "Properties of indoor received signal strength for WLAN location fingerprinting," in Proc. MobiQuitous'04, San Diego, CA, 2004, pp. 14-23.

[17] T. Roos, P. Myllymki, H. Tirri, P. Misikangas, and J. Sievnen, "A probabilistic approach to wlan user location estimation," International Journal of Wireless Information Networks, vol. 9, pp. 155-164, 2002.

[18] A. K. Jain, R. Duin, and J. Mao, "Statistical pattern recognition: A review," IEEE Transactions on Pattern Analysis and Machine Intelligence, vol. 22, pp. 4-37, 2000.

[19] R. Battiti, M. Brunato, and A. Delai, "Optimal wireless access point placement for location-dependent services," Universita di Trento, Dipartimento di Informatica e Telecomunicazioni, Tech. Rep. DIT-03-052, 2003.

[20] Y. Chen, J.-A. Francisco, W. Trappe, and R. Martin, "A practical approach to landmark deployment for indoor localization," in Proc. IEEE SECON'06, 2006, pp. 365-373.

[21] T. T. Soong, Fundamentals of Probability and Statistics for Engineers. Wiley, 2004.

[22] S. M. Kay, Fundamentals of Signal Processing, Volume I: Estimation Theory, 2nd ed., 1993. 